\title{
Glucose transporters 1, 3, 6, and 10 are expressed in gastric cancer and glucose transporter 3 is associated with UICC stage and survival
}

\author{
Hans Anton Schlößer ${ }^{1} \cdot$ Uta Drebber $^{2} \cdot$ Alexander Urbanski $^{1} \cdot$ Simon Haase $^{1}$ • \\ Christoph Baltin $^{1} \cdot$ Felix Berlth $^{1} \cdot$ Susanne Neiß $^{1} \cdot$ Michael von Bergwelt-Baildon $^{4}$. \\ Ulrich Klaus Fetzner $^{1} \cdot$ Ute Warnecke-Eberz $^{1} \cdot$ Elfriede Bollschweiler $^{1}$ • \\ Arnulf Heinrich Hölscher ${ }^{1}$ - Stefan Paul Mönig ${ }^{1}$ - Hakan Alakus ${ }^{1,3}$
}

Received: 27 February 2015/ Accepted: 13 November 2015/Published online: 7 December 2015

(C) The International Gastric Cancer Association and The Japanese Gastric Cancer Association 2015

\begin{abstract}
Background Due to proliferation and increased metabolism, cancer cells have high glucose requirements. The glucose uptake of cells is influenced by a group of membrane proteins denoted the glucose transporter family (Glut-1 to -12). Whereas increased expression and a negative correlation with survival have been described for Glut-1 in several types of cancer, the impact of other glucose transporters on tumor biology is widely unknown. Methods In this retrospective study, gastric cancer specimens of 150 patients who underwent total gastrectomy between 2005 and 2010 were stained for Glut-1, -3, -6, and -10 by immunohistochemistry. Expression of Glut- $1,-3,-6$, and 10 was correlated to prognosis as well as clinical and pathological parameters.

Results Glut-1, Glut-3, Glut-6, and Glut-10 were expressed in 22.0, 66.0, 38.0, and $43.3 \%$ of the analyzed samples. Whereas Glut-1, -6 , and -10 did not show a correlation with prognosis, positive staining for Glut-3 was
\end{abstract}

Stefan Paul Mönig and Hakan Alakus contributed equally to this work.

Hans Anton Schlößer

hans.schloesser@uk-koeln.de

1 Department of General, Visceral and Cancer Surgery, University of Cologne, Kerpener Str. 62, 50937 Cologne, Germany

2 Institute of Pathology, University of Cologne, Cologne, Germany

3 Division of Genome Information Sciences, Department of Pediatrics and Rady Children's Hospital, University of California, San Diego, La Jolla, CA, USA

4 Department of Internal Medicine I, University of Cologne, Cologne, Germany associated with higher UICC stage and inferior prognosis. The mean overall survival was 38.6 months for Glut-3 positive patients, as compared to 51.2 months for Glut-3 negative patients $(p<0.05)$. Coexpression of two or more of the analyzed glucose transporters was correlated to inferior prognosis. Glut-3 and UICC stage were significant prognostic factors in multivariate analysis.

Conclusions All of the analyzed glucose transporters were expressed in a significant proportion of the gastric cancer samples. Glut-3 was associated with higher UICC stage and inferior prognosis. These findings are relevant to therapeutic approaches that target glucose metabolism as well as to imaging using radioactively labeled glucose.

Keywords Gastric cancer - Glucose transporter . Prognosis

\section{Introduction}

As long ago as 1958, Warburg reported increased glucose metabolism in cancer cells. Due to high proliferation and increased metabolism, cancer cells require large amounts of energy, which is mainly obtained via aerobic and anaerobic glycolysis [1]. Positron emission tomography (PET) uses this enhanced glucose uptake of cancerous cells to image various malignancies [2]. The glucose uptake of cells largely depends on the concentration of membrane transport proteins collectively known as as the glucose transporter (Glut) family. These members of the major facilitator superfamily are encoded by SLC2 genes and have been classified into three subsets according to sequence similarity: (I) Glut-1, $-2,-3,-4,-5,-14$; (II) Glut$5,-7,-9,-11$; (III) Glut-6, -8, -10, -12, -13. Glucose transporter proteins have different affinities for glucose and 
remarkably different transport capacities (defined by the half-saturation concentration, $K_{\mathrm{m}}$ ) [3]. Almost every eukaryotic cell expresses at least one glucose transporter, but the subtypes differ largely between tissues and species. As the brain, for example, needs large amounts of glucose, neurons express glucose transporter 3 (Glut-3), which has the lowest $K_{\mathrm{m}}$ [2]. Glut- 1 is the most studied member of the SLC2 family and has been described in several types of cancer. We previously reported that the expression of Glut1 correlates with $18 \mathrm{~F}$-fluorodeoxyglucose uptake and hence the PET image intensity of gastric cancer [4]. Furthermore Glut-1, is a negative prognostic factor for several types of cancer, such as lung cancer, rectal cancer, breast cancer, and esophageal cancer [5-8]. Glut-1 is also associated with an inferior prognosis in gastric cancer [9]. Whereas the impact of Glut-1 in cancer has been studied extensively, the roles of other members of SLC2 in cancer are unknown. Glut-3 is expressed in different kinds of cancer and has been identified as a negative prognostic factor for lung cancer, endometrial cancer, and laryngeal cancer [10-12]. Younes et al. [13] described the expression of Glut-3 in 2/8 gastric cancer samples, and it was not detected in normal gastric mucosa. Glut- 6 to -14 were first described during the Human Genome Project. Thus, data on their physiological function or expression in human tissues are scarce [3], and there are no published data on the expression of Glut- 6 or Glut-10 in cancer. In this study, we analyzed the expression of Glut-3, which has a low $K_{\mathrm{m}}$ for glucose and hence potentially favors glucose uptake in malignant cells, as well as two other transporters with high glucose affinities (Glut-6 and Glut-10) and their potential association with survival, pathological parameters, and expression of Glut-1 in primary gastric cancer.

\section{Materials and methods}

\section{Patient characteristics}

In this retrospective study, specimens from 150 patients with a diagnosis of primary gastric cancer or adenocarcinoma of the gastroesophageal junction (Siewert type II or III) were analyzed. 114 males and 36 females with a mean age of 64.0 years $( \pm 12.5)$ were included. All patients underwent total gastrectomy at the Department of General, Visceral and Cancer Surgery, University of Cologne, Germany between 2006 and 2011. Clinical staging (endoscopy, endoscopic ultrasonography, and CT chest/abdomen) was performed prior to surgery. 69 (46\%) of the included patients received perioperative chemotherapy with etoposide, cyclophosphamide, and fluorouracil according to the MAGIC protocol [14]. The results of histopathological staging according to the UICC 2009 classification and the clinical parameters of the patients are summarized in Table 1. Nine patients were lost to followup. Of the remaining 141 patients, $78(55.3 \%)$ had died by the time that the analyses were performed. Mean follow-up was 33.2 months $( \pm 24.2)$. The present study was conducted according to the guidelines of our local research ethics commission.

\section{Immunohistochemistry}

Paraffin-embedded tumor samples were retrieved from our Institute of Pathology. Gastrectomy specimens were originally processed for pathological diagnosis using standard pathological procedures. The retrieved samples were processed and stained using a Dako EnVision kit (Dako, Hamburg, Germany) according to the manufacturers instructions. Briefly, the slides were first deparaffinized and rehydrated. Antigen retrieval was performed with microwave incubation $(3 \times 5 \mathrm{~min}, 600 \mathrm{~W})$ in citrate buffer (pH 6.0). After treatment with peroxidase blocking solution and Tris buffer, the slides were incubated with primary antibodies $(1: 100)$ at $4{ }^{\circ} \mathrm{C}$ overnight. Appropriate positive (breast cancer and erythrocytes for Glut-1, human testicle for Glut-3 and Glut-10, small intestine for Glut-6) and negative controls for Glut-3, Glut-6, and Glut-10 were included in every staining procedure. After $30 \mathrm{~min}$ of incubation with the horseradish peroxidase conjugated secondary antibody (EnVision kit), AEC was added for

Table 1 Patient characteristics

\begin{tabular}{ll}
\hline Age & $64( \pm 12.5)$ years \\
Sex & \\
Male & $114(76.0 \%)$ \\
Female & $36(24.0 \%)$ \\
UICC stage & \\
St. I & $55(36.7 \%)$ \\
St. II & $45(30.0 \%)$ \\
St. III & $25(16.7 \%)$ \\
St. IV & $25(16.7 \%)$ \\
Metastasis & \\
Yes & $25(16.7 \%)$ \\
No & $125(83.3 \%)$ \\
Resection margin & \\
R0 & $137(91.3 \%)$ \\
R1 & $13(8.7 \%)$ \\
Chemotherapy & \\
ECX (MAGIC) & $69(46.0 \%)$ \\
None & $81(54.0 \%)$ \\
Lauren classification & \\
Diffuse type & $90(60.0 \%)$ \\
Intestinal type & $60(40.0 \%)$ \\
\hline
\end{tabular}


15,30 , and 15 min for Glut-3, -6 , and -10 , respectively. Finally, the slides were stained with hemalum and covered.

Expression of glucose transporters 1, 3, 6, and 10 was semiquantitatively assessed by an experienced pathologist (U.D.) who was blinded to clinical parameters. To make sure that the analyzed blocks contained sufficient amounts of tumor tissue, the hematoxylin and eosin staining of each block was examined. Analyses of glucose transporter expression were performed on whole tissue sections with representative amounts of gastric adenocarcinoma tissue. The tumor areas were examined, and the expression of Glut-1, $-3,-6$, or -10 was scored according to the percentage of positive tumor cells $(0=$ absent or very weak staining, $1=$ clear positive staining of at least $10 \%$ of tumor cells).

\section{Data analyses}

The expression of each glucose transporter was correlated to clinical and pathological parameters. SPSS version 20 (IBM Corp.) was used for statistical analysis. The chisquare test, Kaplan-Meier survival analysis, and Cox regression analysis were used as appropriate. $p$ values $<0.05$ were considered significant.

\section{Results}

\section{Expression of glucose transporter 1 in gastric cancer is associated with an inferior prognosis}

Glut-1 was expressed on tumor cells in $22.0 \%$ of the analyzed samples (33/150, Table 2$)$. The staining pattern was mainly membranous (Fig. 1a). Expression of Glut-1 was correlated to intestinal type according to Lauren. The frequency was $41.7 \%(25 / 60)$ in intestinal type cancer compared to $8.9 \%(8 / 90)$ in diffuse-type cancer $(p \leq 0.001)$. Glut-1 was not correlated to UICC stage (10.9\% (6/55), $31.1 \%(14 / 45), 20.0 \%(5 / 25)$, and $32.0 \%$ $(8 / 25)$ in stage I, II, III, and IV, respectively, $p=0.054)$. Glut-1 was correlated to lower $\mathrm{T}$ stage and was independent of $\mathrm{N}$ stage (Table 2) and $\mathrm{M}$ stage (data not shown). Kaplan-Meier survival analysis revealed an inferior prognosis for patients expressing Glut-1 on primary cancer cells [mean overall survival was 45.6 months $( \pm 3.2)$ for Glut-1 negative patients $(n=110)$ and $34.2( \pm 5.8)$ months for Glut-1 positive patients $(n=31)$ ], but this difference was not significant ( $p=0.078$, Fig. 2a).

\section{Glucose transporter 3 is associated with UICC stage and prognosis of gastric cancer}

Immunohistochemical analyses of full-section slides revealed that Glut-3 was expressed in $66.0 \%(99 / 150)$ of the tumor samples analyzed. The staining pattern was membranous and cytoplasmic. Positive samples showed mainly homogeneous staining of the tumor areas, whereas adjacent normal tissue was negative in almost every sample analyzed (Fig. 1b). The expression of Glut-3 in gastric cancer is associated with UICC stage: whereas only $52.7 \%$ (29/55) of stage I gastric cancer samples showed expression of Glut-3, the percentage was $77.8 \%(35 / 45), 60 \%$ $(15 / 25)$, and $80 \%(20 / 25)$ in stage II, III, and IV, respectively ( $p=0.021$, Table 2 ). Glut-3 expression was correlated to intestinal type according to Lauren [78.3\% (47/60) of intestinal type samples were Glut-3 positive as compared to $57.8 \%$ (52/90) of diffuse-type samples, $p=0.009]$ and was not correlated to $\mathrm{T}$ stage, $\mathrm{N}$ stage, or metastasis $(p=0.33,0.054$, and 0.164 , respectively). Kaplan-Meier survival analysis revealed an inferior prognosis for gastric cancer patients who showed expression of Glut-3 in primary tumor samples. Mean overall survival was 51.2 months $( \pm 4.6)$ for Glut-3 negative patients $(n=48)$ as compared to 38.6 months $( \pm 3.4)$ for Glut-3 positive patients ( $n=93, p=0.028$, Fig. $2 b$ ).

\section{Glucose transporter 6 is expressed in gastric cancer}

Glucose transporter 6 was expressed in $38.0 \%(57 / 150)$ of the analyzed samples. Similar to Glut-3, tumor areas of positive samples showed homogeneous cytoplasmic and membranous staining, whereas normal gastric mucosa usually did not express Glut-6 (Fig. 2c). Associations of Glut-6 expression with clinical and pathological parameters were analyzed. Glut-6 expression was correlated to intestinal type according to Lauren [48.3\% (29/60) of intestinal type cancers and $31.1 \%$ (28/90) of diffuse-type cancers expressed Glut-6, $p=0.033$ ]. Whereas UICC stages I, II, and III revealed remarkable fractions of Glut-6 positive tumors, only $16 \%(4 / 25)$ of the tumor areas in UICC stage IV patients were found to be positive for Glut$6(p=0.030$, Table 2). Expression of Glut-6 was not correlated to $\mathrm{T}(p=0.399)$ or $\mathrm{N}(p=0.961)$ stage. Finally, expression of glucose transporter 6 was not correlated to prognosis of gastric cancer (mean overall survival of $42.6( \pm 4.4)$ months vs. $43.4( \pm 3.6)$ months in Glut- 6 positive $(n=53)$ and negative $(n=88)$ patients, respectively, $p=0.898$; Fig. $2 c)$. 


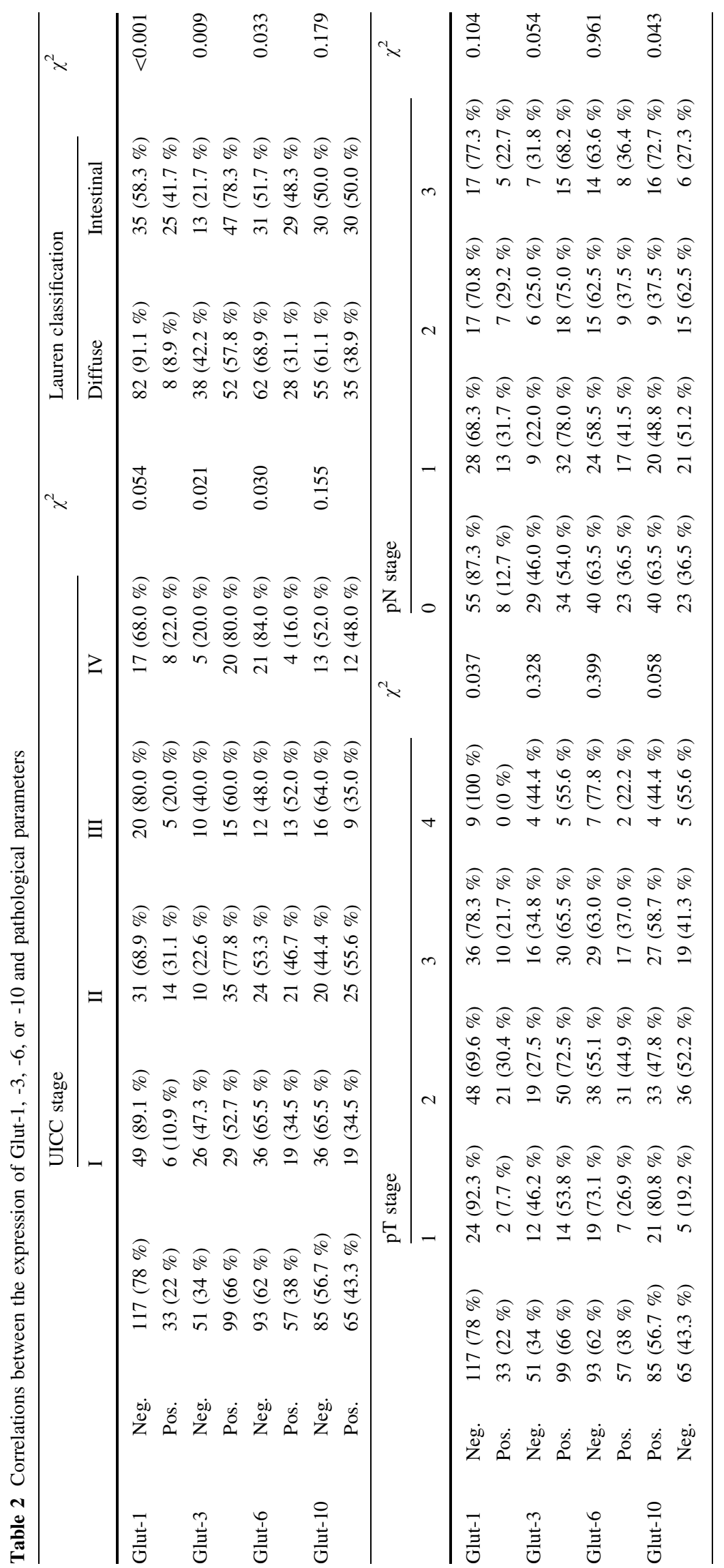


Fig. 1 Representative images showing immunohistochemical staining of primary gastric cancer samples.

a Immunohistochemistry showing the typical membranous staining pattern of glucose transporter 1 (DAB, $\times 20)$. b Immunohistochemical staining of Glut-3 showing strong expression in the tumor area, whereas adjacent normal tissue is negative (DAB, $\times 10)$. c Representative staining of an intestinal type gastric cancer with Glut-6 (DAB, ×40). d Expression of Glut-10 in gastric cancer using immunohistochemistry (DAB, $\times 40$ )
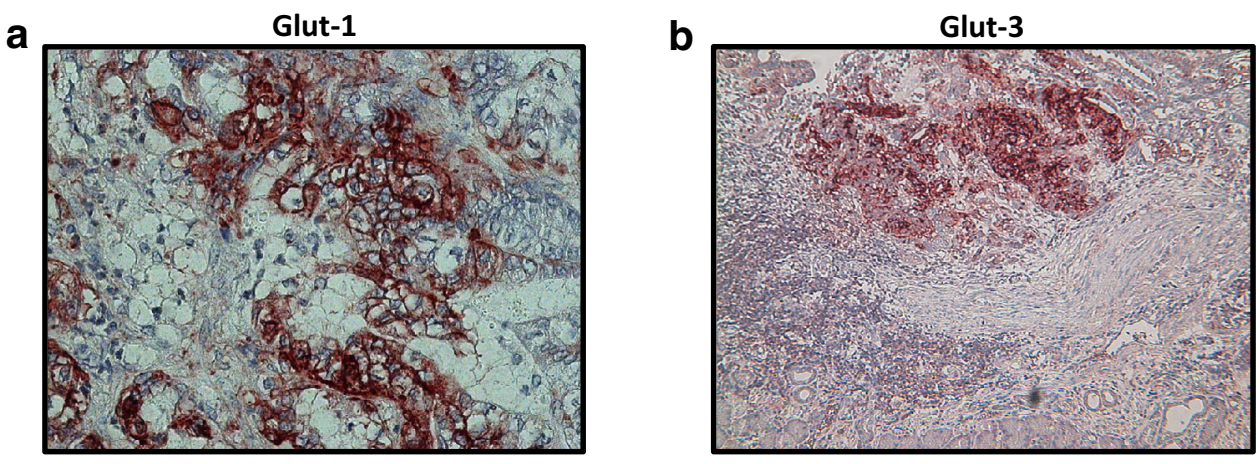

C

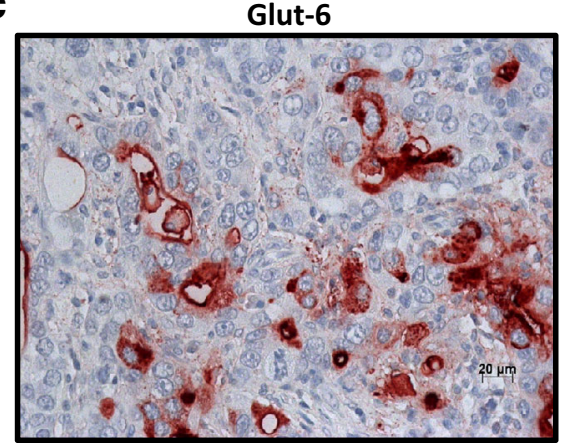

d

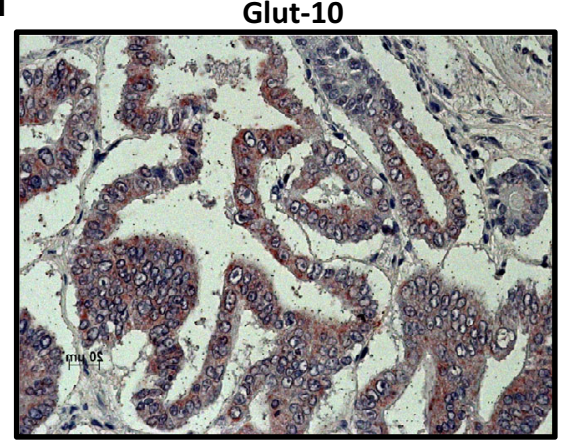

\section{Expression of glucose transporter 10 in gastric cancer}

Regarding the expression of Glut-10 in gastric cancer, our analysis revealed positivity in $43.3 \%(65 / 150)$ of the analyzed samples with a cytoplasmic staining profile, whereas adjacent normal tissue was negative (Fig. 2d). A chi-square test did not show a significant correlation between Glut-10 expression and UICC stage $(p=0.16)$. The exact expression of Glut-10 across the UICC stages was $34.5 \%(19 / 55), 55.6 \%(25 / 45), 36.0 \%$ (9/25), and $48.0 \%(12 / 25)$ in stages I, II, III, and IV, respectively. Furthermore, Glut-10 expression was not correlated to the Lauren classification (50.0\% (30/60) of intestinal type and $38.9 \%(35 / 90)$ of diffuse-type cancers expressed Glut-10, $p=0.179)$, T stage $(p=0.058)$ or metastasis $(p=0.606)$ and correlated to $\mathrm{N}$ stage $(p=0.043)$. The mean overall survival was $45.4( \pm 3.7)$ months for Glut-10 negative patients $(n=81)$ compared to $40.3( \pm 4.2)$ months in Glut10 positive patients $(n=60)$, and this difference was not statistically significant ( $p=0.34$, Fig. $2 d)$.

\section{$53.3 \%$ of the analyzed samples expressed more than one glucose transporter}

$83.3 \%(125 / 150)$ of the analyzed gastric cancer samples were positive for at least one glucose transporter protein. Coexpression of 2-4 glucose transporters was observed in $53.3 \%(80 / 150)$ of the analyzed tumors (Fig. 3). Analyses of correlations between the expression of the examined glucose transporter proteins using Pearson's chi-square test revealed correlations between Glut-1 and Glut-10 $(p=0.02)$, Glut-3 and Glut-6 $(p=0.023)$, Glut-3 and Glut-10 $(p=0.002)$, and between Glut-6 and Glut-10 $(p=0.005)$. The data relating to coexpression are shown in Fig. 3. Expression of $>1$ glucose transporter was correlated to higher pT stage, pN stage, UICC stage, and intestinal type according to Lauren $(p=0.008,0.034$, 0.013 , and 0.008 , respectively). Kaplan-Meier analysis revealed inferior survival of patients expressing more than one glucose transporter $[36.8( \pm 3.7)$ months vs. 49.7 ( \pm 4.1$)$ months; $p=0.02$; Fig. 3c]. In multivariate analysis, UICC stage and expression of Glut-3 were the only significant parameters (Table 3).

\section{Discussion}

Increased glucose uptake is a well-known feature of cancer cells, and is widely used to image tumors using radioactively labeled glucose in positron emission tomography, which is, for example, part of the diagnostic standard in lung cancer $[4,15]$. The glucose uptake capacity of cells largely depends on the expression of glucose transporters, which transport glucose across membranes. The increased glucose requirements of malignant cells were initially described by Otto Warburg more than 50 years ago, and further research on cancer metabolism since then has lent 

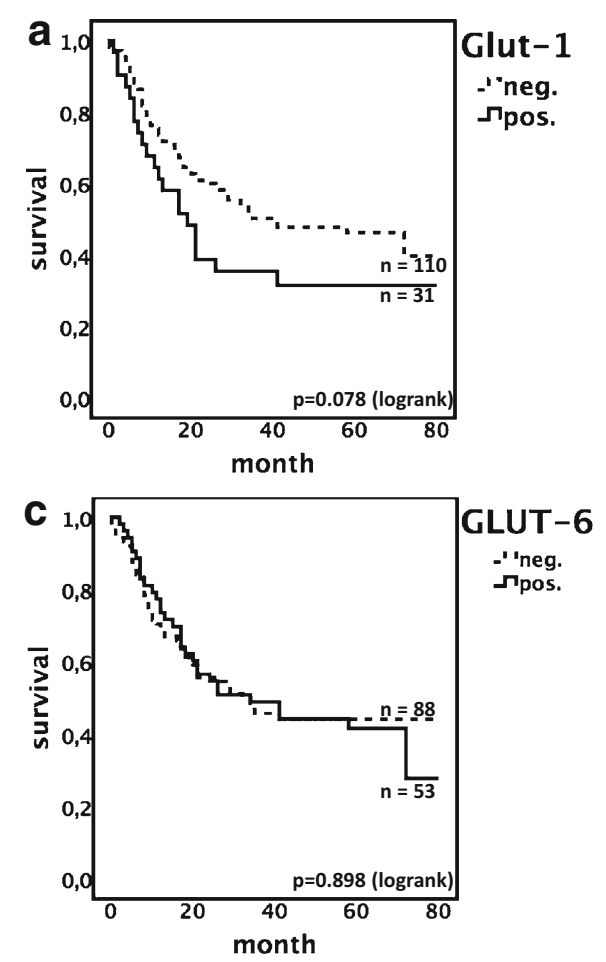

Fig. 2 Kaplan-Meier survival analyses of Glut-1, -3, -6, and -10 . a The mean overall survival for patients expressing Glut-1 was 34.2 months as compared to 45.6 months for negative patients, but this difference did not reach statistical significance in the analyzed cohort $(p=0.078, n=141)$. b Kaplan-Meier survival analysis showing an inferior prognosis for Glut-3 positive patients (mean overall survival of 38.6 months compared to 51.2 in Glut- 3 negative patients, $p=0.028, n=141$ ). c Glut- 6 was expressed in about one-

support to this hypothesis, as increased expression of glucose transporters has been shown for several types of cancer [1]. Several other molecules linked to glucose metabolism (e.g., hypoxia inducible factor $1 \alpha$ and pyruvate dehydrogenase kinase 1) contribute to cancer progression $[16,17]$. Expression of pyruvate dehydrogenase kinase 1 is, for example, correlated to an inferior prognosis in several malignancies, including gastric cancer [18-20]. Additionally, hypoxia-a common feature in the tumor microenvironment-is closely linked to glucose metabolism. Hypoxia inducible factor $1 \alpha$ can, for example, induce the expression of Glut-1 and Glut-3 [21]. For Glut-1, the beststudied member of the glucose transporter family, increased expression has been shown in several different entities such as colorectal cancer [22], head and neck cancer [23], lung cancer [24], and ovarian carcinoma, and it is often associated with an inferior prognosis [22]. For gastric cancer, Kawamura et al. described the expression of Glut- 1 in $29.8 \%$ of the samples they analyzed. Glut- 1 was a negative prognostic factor in this cohort of 617 patients. Although Griffiths et al. [9, 25] could not confirm the prognostic relevance in their European cohort, Glut-1 was

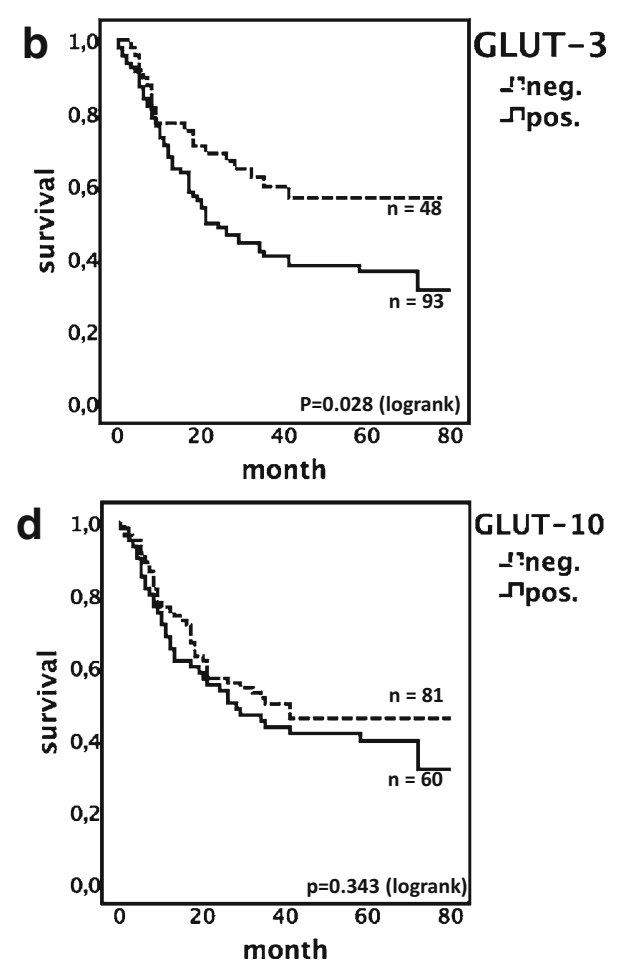

third of the samples analyzed, but positivity was not correlated to survival (mean overall survival was 42.6 months for Glut-6 positive patients compared to 43.0 months for Glut- 6 negative patients, $p=0.96, n=140$ ). d Expression of Glut-10 was not correlated to prognosis in this cohort (mean overall survival 40.3 months for patients with tumors expressing Glut-10 as compared to 45.4 months for Glut-10 negative patients, $p=0.343, n=141$ )

associated with tumor invasiveness in their analysis. In our analysis, patients with tumors expressing Glut-1 had an inferior prognosis, but the difference was not significant (Fig. $2 \mathrm{a}, p=0.078$ ). The widespread expression of Glut-1 in many normal tissues complicates the translation of these findings into clinical applications, as it potentially leads to severe side effects. The Glut-1 inhibitor STK-31 efficiently inhibited glucose uptake into renal cell carcinoma xenografts and markedly reduced tumor growth in two animal models of RCC. Although this agent also inhibits glucose uptake in human erythrocytes, it did not cause hemolysis. As it did not affect normal tissues in treated animals, it may represent a therapeutic option to target glucose metabolism in human cancer [21]. Regardless of these positive results, the widespread expression of Glut-1 could be a limiting factor in its clinical application, and identifying other members of the glucose transporter family with greater specificity for tumor tissue could facilitate their translation into clinical use.

Due to these limitations, we set out to identify Glut-3, Glut-6, and Glut-10 in gastric cancer, as these proteins show limited expression in normal tissues and high glucose 


\begin{tabular}{|c|c|c|c|c|c|c|c|}
\hline & & & $\mathbf{0}$ & 1 & 2 & 3 & 4 \\
\hline \multicolumn{3}{|c|}{ No. expressed Glut's } & $25(16.7 \%)$ & $45(30 \%)$ & $41(27.3 \%$ & $29(19.3 \%)$ & $10(6.7 \%)$ \\
\hline \multirow{4}{*}{\multicolumn{4}{|c|}{ coexpressions }} & \multicolumn{4}{|c|}{ Glut-3 } \\
\hline & & & & \multicolumn{2}{|c|}{ neg. (51) } & \multicolumn{2}{|c|}{ pos. (99) } \\
\hline & & & & \multicolumn{2}{|c|}{ Glut-1 } & \multicolumn{2}{|c|}{ Glut-1 } \\
\hline & & & & neg. (44) & pos. (7) & neg. (73) & pos. (26) \\
\hline \multirow{4}{*}{ Glut-10 } & \multirow{2}{*}{ neg (85) } & \multirow{2}{*}{ Glut-6 } & neg (61) & 25 & 5 & 26 & 5 \\
\hline & & & pos (24) & 6 & 2 & 15 & 1 \\
\hline & \multirow{2}{*}{ pos (65) } & \multirow{2}{*}{ Glut-6 } & neg (32) & 8 & 0 & 14 & 10 \\
\hline & & & pos (33) & 5 & 0 & 18 & 10 \\
\hline
\end{tabular}

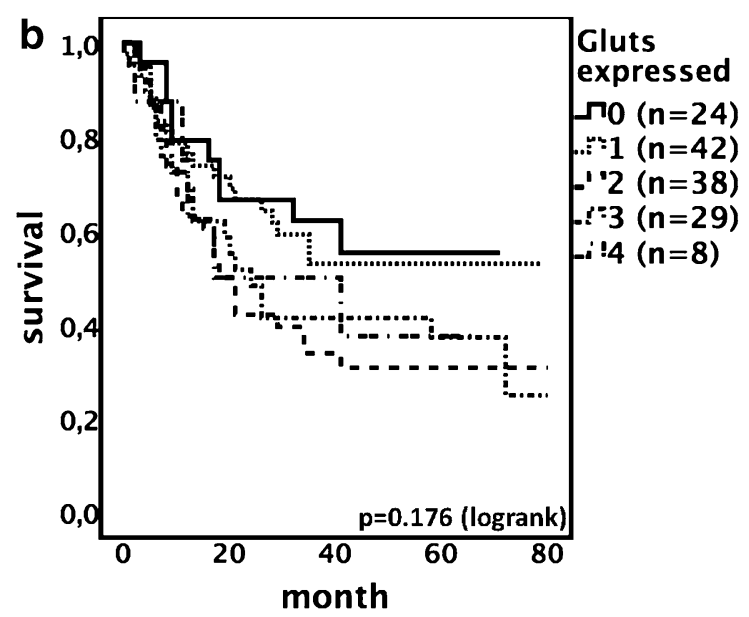

Fig. 3 Coexpression of glucose transporters 1, 3, 6, and 10. a Data on the coexpression of the analyzed glucose transporters. At least one Glut was expressed in $83.3 \%$ of the samples analyzed. b KaplanMeier plot showing the association between the number of expressed glucose transporters and survival $(p=0.176)$. c Mean overall

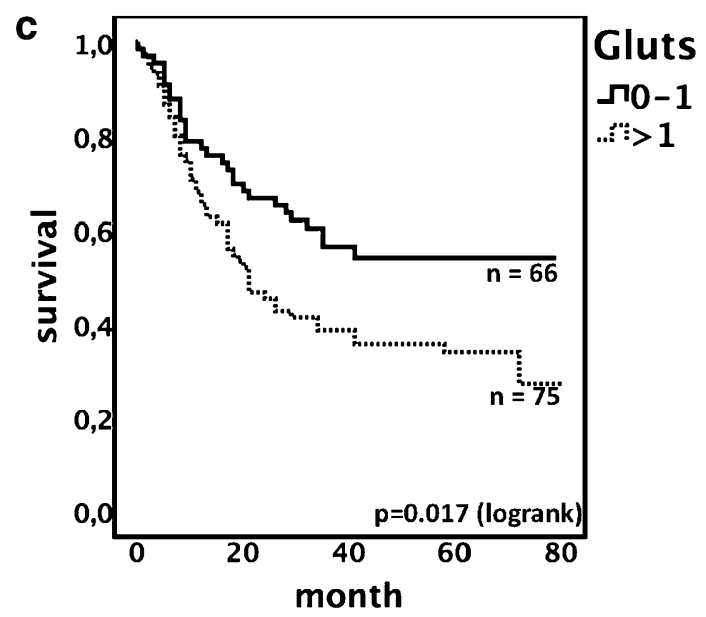

survival of patients expressing more than one glucose transporter was 36.8 months as compared to 50.1 months for patients expressing one or none of the analyzed glucose transporters $(p=0.014$, $n=141$ )

Table 3 Multivariate analysis

\begin{tabular}{lllll}
\hline Variable & & Hazard ratio & $95 \%$ CI & $p$ value \\
\hline Glut-1 & Pos/neg & 1.731 & $0.988-3.033$ & 0.055 \\
Glut-3 & Pos/neg & 1.852 & $1.044-3.284$ & 0.035 \\
Glut-6 & Pos/neg & 0.917 & $0.551-1.525$ & 0.738 \\
Glut-10 & Pos/neg & 1.179 & $0.719-1.935$ & 0.514 \\
UICC stage & III + IV/I + II & 4.853 & $2.848-8.270$ & 0.000 \\
Lauren & Intestinal/diffuse & 0.683 & $0.398-1.172$ & 0.167 \\
Chemotherapy & Yes/no & 1.527 & $0.907-2.573$ & 0.112 \\
$R$ & $1 / 0$ & 1.855 & $0.937-3.671$ & 0.076 \\
Sex & Female/male & 0.585 & $0.325-1.052$ & 0.073 \\
Age & $>70 /<70$ & 1.575 & $0.889-2.792$ & 0.119 \\
\hline
\end{tabular}

affinity has been observed for each of them [26]. Glut-3 was expressed in $66 \%$ of the gastric cancer samples we analyzed. Glut-3 is preferentially expressed in tissues with high energy needs. The strong expression in neurons and embryonic cells demonstrates the importance of Glut-3 for glucose homeostasis in vulnerable tissues [27]. In the context of malignant disease, it theoretically represents a stronger positive selection factor than Glut-1, as its glucose affinity is far higher [3]. Nevertheless, most studies have focused on Glut-1, and there is very limited knowledge of the role of Glut-3 in cancer. It has been detected as a negative prognostic factor in non-small cell lung cancer, head and neck cancer, and thyroid cancer [11, 12, 28, 29]. Younes et al. described the expression of Glut-3 in 2/8 of 
the gastric cancer samples they analyzed [13]. We were able to confirm the expression of Glut-3 in our large cohort of gastric cancer patients. In the present study, positive staining for Glut-3 was correlated with higher UICC stage and inferior prognosis (Table 2, Fig. 2b). Furthermore, it was a significant prognostic factor in multivariate analysis (Table 3). The hazard ratio of 1.85 was rather low but similar to previous results regarding Glut-3 expression in head and neck cancer or Glut-1 in gastric cancer or esophageal cancer $[9,30]$. To the best of our knowledge, this is the first study to correlate clinical and pathological parameters with the expression of Glut-3 in gastric cancer. The other glucose transporters investigated (Glut- 6 and Glut-10) were also expressed in a significant proportion of the samples analyzed (38.0 and $43.3 \%$ for Glut- 6 and Glut-10, respectively) but did not show a significant correlation to prognosis. Still, our analysis of Glut-6 and Glut10 in gastric cancer represents the first data to show the expression of Glut-10 in human cancer and the first to show the expression of Glut- 6 in gastric cancer. Expression of Glut- 6 has previously been described in breast and pancreatic cancer as part of a screening analysis that included small cohorts $(<10)$ of 14 tumor entities [31]. Whereas the authors of that study described the expression of Glut- 6 in $2 / 2$ of the normal stomach tissues they analyzed, they did not see positivity in gastric cancer (0/6). Those results contrast with our results, where normal tissue was hardly ever positive but about one-third $(38.0 \%)$ of the tumor samples analyzed expressed Glut-6. However, as Glut-6 and Glut-10 did not show any prognostic relevance and were expressed in less than half of the samples analyzed, we assume that the strong expression of Glut- 3 is the most important finding of this study. Due to its higher glucose affinity and broader expression in gastric cancer, it may be of greater clinical relevance than the well-established Glut1 , which is expressed in $<30 \%$ of gastric cancer samples [9]. Furthermore, both proteins seem to have a similar relevance in FDG-PET imaging [32].

In conclusion, our study demonstrates that glucose transporters other than Glut-1 are highly expressed in primary gastric cancer and may play a crucial role in glucose homeostasis of gastric cancer cells. Glut-3, which is negatively correlated with survival, is an especially interesting target in this cancer (for which there are very limited therapeutic options). Furthermore, the broad expression of the analyzed glucose transporter proteins has interesting implications for positron emission tomography. Acknowledgments The German "Nolting Stiftung" supported this
work.

\section{Compliance with ethical standards}

Conflict of interest The authors declare no conflict of interest.

\section{References}

1. Koppenol WH, Bounds PL, Dang CV. Otto Warburg's contributions to current concepts of cancer metabolism. Nat Rev Cancer. 2011;11:325-37.

2. Macheda ML, Rogers S, Best JD. Molecular and cellular regulation of glucose transporter (GLUT) proteins in cancer. J Cell Physiol. 2005;202:654-62.

3. Mueckler M, Thorens B. The SLC2 (GLUT) family of membrane transporters. Mol Aspects Med. 2013;34:121-38.

4. Alakus H, Batur M, Schmidt M, Drebber U, Baldus SE, Vallböhmer D, et al. Variable 18F-fluorodeoxyglucose uptake in gastric cancer is associated with different levels of GLUT-1 expression. Nucl Med Commun. 2010;31:532-8.

5. Meijer TWH, Schuurbiers OCJ, Kaanders JHAM, Looijen-Salamon MG, de Geus-Oei LF, Verhagen AFTM, et al. Differences in metabolism between adeno- and squamous cell non-small cell lung carcinomas: spatial distribution and prognostic value of GLUT1 and MCT4. Lung Cancer. 2012;76:316-23.

6. Saigusa S, Toiyama Y, Tanaka K, Okugawa Y, Fujikawa H, Matsushita K, et al. Prognostic significance of glucose transporter-1 (GLUT1) gene expression in rectal cancer after preoperative chemoradiotherapy. Surg Today. 2012;42:460-9.

7. Pinheiro C, Sousa B, Albergaria A, Paredes J, Dufloth R, Vieira D, et al. GLUT1 and CAIX expression profiles in breast cancer correlate with adverse prognostic factors and MCT1 overexpression. Histol Histopathol. 2011;26:1279-86.

8. Tohma T, Okazumi S, Makino H, Cho A, Mochizuki R, Shuto K, et al. Overexpression of glucose transporter 1 in esophageal squamous cell carcinomas: a marker for poor prognosis. Dis Esophagus. 2005;18:185-9.

9. Kawamura T, Kusakabe T, Sugino T, Watanabe K, Fukuda T, Nashimoto A, et al. Expression of glucose transporter-1 in human gastric carcinoma: association with tumor aggressiveness, metastasis, and patient survival. Cancer. 2001;92:634-41.

10. Krzeslak A, Wojcik-Krowiranda K, Forma E, Jozwiak P, Romanowicz H, Bienkiewicz A, et al. Expression of GLUT1 and GLUT3 glucose transporters in endometrial and breast cancers. Pathol Oncol Res. 2012;18:721-8.

11. Baer S, Casaubon L, Schwartz MR, Marcogliese A, Younes M. Glut 3 expression in biopsy specimens of laryngeal carcinoma is associated with poor survival. Laryngoscope. 2002;112:393-6.

12. Younes M, Brown RW, Stephenson M, Gondo M, Cagle PT. Overexpression of Glut1 and Glut3 in stage I nonsmall cell lung carcinoma is associated with poor survival. Cancer. 1997;80:1046-51.

13. Younes M, Lechago LV, Somoano JR, Mosharaf M, Lechago J. Immunohistochemical detection of Glut 3 in human tumors and normal tissues. Anticancer Res. 1997;17:2747-50.

14. Cunningham D, Allum WH, Stenning SP, Thompson JN, Van de Velde CJH, Nicolson M, et al. Perioperative chemotherapy versus surgery alone for resectable gastroesophageal cancer. N Engl J Med. 2006;355:11-20.

15. Kobayashi M, Kaida H, Kawahara A, Hattori S, Kurata S, Hayakawa M, et al. The relationship between GLUT-1 and vascular endothelial growth factor expression and 18F-FDG uptake in esophageal squamous cell cancer patients. Clin Nucl Med. 2012;37:447-52.

16. Vasseur S, Afzal S, Tardivel-Lacombe J, Park DS, Iovanna JL, Mak TW. DJ-1/PARK7 is an important mediator of hypoxia-induced cellular responses. Proc Natl Acad Sci USA. 2009;106:1111-6.

17. Kaelin WG, Thompson CB. Q\&A: cancer: clues from cell metabolism. Nature. 2010;465:562-4.

18. Wigfield SM, Winter SC, Giatromanolaki A, Taylor J, Koukourakis ML, Harris AL. PDK-1 regulates lactate production in 
hypoxia and is associated with poor prognosis in head and neck squamous cancer. Br J Cancer. 2008;98:1975-84.

19. Baumunk D, Reichelt U, Hildebrandt J, Krause H, Ebbing J, Cash $\mathrm{H}$, et al. Expression parameters of the metabolic pathway genes pyruvate dehydrogenase kinase-1 (PDK-1) and DJ-1/PARK7 in renal cell carcinoma (RCC). World J Urol. 2013;31:1191-6.

20. Hur H, Xuan Y, Kim YB, Lee G, Shim W, Yun J, et al. Expression of pyruvate dehydrogenase kinase-1 in gastric cancer as a potential therapeutic target. Int J Oncol. 2013;42:44-54.

21. Chan DA, Sutphin PD, Nguyen P, Turcotte S, Lai EW, Banh A, et al. Targeting GLUT1 and the Warburg effect in renal cell carcinoma by chemical synthetic lethality. Sci Transl Med. 2011;3:94ra70.

22. Shen Y-M, Arbman G, Olsson B, Sun X-F. Overexpression of GLUT1 in colorectal cancer is independently associated with poor prognosis. Int J Biol Mark. 2011;26:166-72.

23. Eckert AW, Kappler M, Schubert J, Taubert H. Correlation of expression of hypoxia-related proteins with prognosis in oral squamous cell carcinoma patients. Oral Maxillofac Surg. 2012;16:189-96.

24. Sasaki H, Shitara M, Yokota K, Hikosaka Y, Moriyama S, Yano M, et al. Overexpression of GLUT1 correlates with Kras mutations in lung carcinomas. Mol Med Rep. 2012;5:599-602.

25. Griffiths EA, Pritchard SA, McGrath SM, Valentine HR, Price PM, Welch IM, et al. Hypoxia-associated markers in gastric carcinogenesis and HIF-2alpha in gastric and gastro-oesophageal cancer prognosis. Br J Cancer. 2008;98:965-73.
26. Wood IS, Trayhurn P. Glucose transporters (GLUT and SGLT): expanded families of sugar transport proteins. $\mathrm{Br} \mathrm{J}$ Nutr. 2003;89:3-9.

27. Simpson IA, Dwyer D, Malide D, Moley KH, Travis A, Vannucci SJ. The facilitative glucose transporter GLUT3: 20 years of distinction. Am J Physiol Endocrinol Metab. 2008;295:E242-53.

28. Schönberger J, Rüschoff J, Grimm D, Marienhagen J, Rümmele $\mathrm{P}$, Meyringer R, et al. Glucose transporter 1 gene expression is related to thyroid neoplasms with an unfavorable prognosis: an immunohistochemical study. Thyroid. 2002;12:747-54.

29. Ayala FRR, Rocha RM, Carvalho KC, Carvalho AL, da Cunha IW, Lourenço SV, et al. GLUT1 and GLUT3 as potential prognostic markers for oral squamous cell carcinoma. Molecules. 2010;15:2374-87.

30. Sawayama H, Ishimoto T, Watanabe M, Yoshida N, Baba Y, Sugihara $\mathrm{H}$, et al. High expression of glucose transporter 1 on primary lesions of esophageal squamous cell carcinoma is associated with hematogenous recurrence. Ann Surg Oncol. 2014;21:1756-62.

31. Godoy A, Ulloa V, Rodríguez F, Reinicke K, Yañez AJ, García MDLA, et al. Differential subcellular distribution of glucose transporters GLUT1-6 and GLUT9 in human cancer: ultrastructural localization of GLUT1 and GLUT5 in breast tumor tissues. J Cell Physiol. 2006;207:614-27.

32. de Geus-Oei L-F, van Krieken JHJM, Aliredjo RP, Krabbe PFM, Frielink C, Verhagen AFT, et al. Biological correlates of FDG uptake in non-small cell lung cancer. Lung Cancer. 2007;55:79-87. 